\title{
Integrable Time-Discretisation of the Ruijsenaars-Schneider Model $^{1}$
}

\author{
F.W. Nijhoff \\ Department of Applied Mathematical Studies \\ The University of Leeds, Leeds LS2 9JT, UK \\ O. Ragnisco \\ Dipartimento di Fisica E. Amaldi, Università di Roma III \\ and Istituto di Fisica Nucleare, Sezione di Roma \\ P. le Aldo Moro 2, 00185 Roma, Italy \\ V.B. Kuznetsov \\ Faculteit voor Wiskunde en Informatica, Universiteit van Amsterdam ${ }^{2,3}$ \\ Plantage Muidergracht 24, 1018 TV Amsterdam, The Netherlands
}

\begin{abstract}
An exactly integrable symplectic correspondence is derived which in a continuum limit leads to the equations of motion of the relativistic generalization of the Calogero-Moser system, that was introduced for the first time by Ruijsenaars and Schneider. For the discrete-time model the equations of motion take the form of Bethe Ansatz equations for the inhomogeneous spin- $\frac{1}{2}$ Heisenberg magnet. We present a Lax pair, the symplectic structure and prove the involutivity of the invariants. Exact solutions are investigated in the rational and hyperbolic (trigonometric) limits of the system that is given in terms of elliptic functions. These solutions are connected with discrete soliton equations. The results obtained allow us to consider the Bethe Ansatz equations as ones giving an integrable symplectic correspondence mixing the parameters of the quantum integrable system and the parameters of the corresponding Bethe wavefunction.
\end{abstract}

\footnotetext{
${ }^{1}$ Report 94-27, Mathematical preprint series, University of Amsterdam; hep-th/9412170.

${ }^{2}$ Supported by the Nederlandse Organisatie voor Wetenschappelijk Onderzoek (NWO).

${ }^{3}$ On leave from Department of Mathematical and Computational Physics, Institute of Physics, St. Petersburg University, St. Petersburg 198904, Russia.
} 


\section{Introduction}

In some previous papers, [1, 2], cf. also [3], an exact time-discretization of the famous Calogero-Moser (CM) model, [4]-[7], was introduced and investigated. The discrete model is an integrable symplectic correspondence, (for a definition cf. [8]), that in a well-defined continuum limit yields the classical equations of motion of the CM system円. A few years ago Ruijsenaars and Schneider introduced in [10], cf. also [11, 12], a relativistic variant of the CM model, which is a parameter-deformation of the original model. The equations of motion of this system in its generic (elliptic) form read

$$
\ddot{q}_{i}=\sum_{j \neq i} \dot{q}_{i} \dot{q}_{j} v\left(q_{i}-q_{j}\right), \quad i=1, \ldots, N
$$

where the potential $v(x)$ is given by

$$
v(x)=\frac{\wp^{\prime}(x)}{\wp(\lambda)-\wp(x)},
$$

in which $\wp(x)=\wp\left(x \mid \omega_{1}, \omega_{2}\right)$ is the Weierstrass P-function, $2 \omega_{1,2}$ being a pair of periods. (These are the equations of motion in the form given by Bruschi and Calogero in [13]). This multi-particle model is also integrable, and carries a representation of the Poincaré algebra in two dimensions. Moreover, a large number of the characteristics of the CM model are generalized in a natural way to the relativistic case, such as the existence of a Lax pair, a sufficient number of integrals of the motion in involution, and exact solution schemes in special cases.

In view of the results in [1] -[3], a natural question to ask is whether there exists a time-discrete version of the model (1.1). We have to remark that on the quantum level the transition from the usual CM system to its relativistic counterpart, already amounts to a discretization (or $q$-deformation). In fact, the relevant operators in the quantum relativistic model are commuting difference operators, rather than differential operators, cf. [14, 15]. In view of the importance of quantum Calogero-Moser type of models in the context of representation theory, and in particular in connection with the KnizhnikZamolodchikov (KZ) equations as has been revealed by a large amount of recent work, cf.

\footnotetext{
${ }^{1}$ It should be noted that the discrete CM model can be inferred also from the Bäcklund transformations for the continuous CM model, that were presented in [9].
} 
e.g. [16]-[18], these difference operators yield new interesting connections with $q$-special functions.

In this paper we will introduce a discrete-time version of the model (1.1), in the form of an integrable symplectic correspondence (i.e. multi-valued map) which goes in a continuum limit to the original model. This amounts to one more parameter-deformation of the CM model: apart from the "spatial" discretisation (encoded in the parameter $\lambda)$, the discretisation of time constitutes another deformation where the finite step-size in time enters as the new parameter. The construction is based on an Ansatz for a Lax pair, together with an elliptic version of the Lagrange interpolation formula. We will demonstrate the integrability of this mapping along the lines of ref. [8, 19], i.e. integrability in the sense of Liouville. In contrast to the previous paper, [2], where the particle model is related to pole solutions of discrete soliton equations, in particular of the lattice Kadomtsev-Petviashvili (KP) equation, the relativistic case is related to soliton solutions of this type of discrete equations. Finally, we point out the intriguing resemblance between the equations of motion of the discrete particle model and Bethe Ansatz equations for integrable spin- $\frac{1}{2}$ quantum chains of the XYZ Heisenberg model (see [36, 39]).

\section{Derivation of the Model}

We will start from an Ansatz for a Lax pair of the form

$$
\begin{aligned}
L_{\kappa} & =\sum_{i, j=1}^{N} h_{i} h_{j} \Phi_{\kappa}\left(q_{i}-q_{j}+\lambda\right) e_{i j}, \\
M_{\kappa} & =\sum_{i, j} \widetilde{h}_{i} h_{j} \Phi_{\kappa}\left(\widetilde{q}_{i}-q_{j}+\lambda\right) e_{i j} .
\end{aligned}
$$

In (2.1) the $q_{i}$ denote the particle positions, and the $h_{i}$ are auxiliary variables which we specify later. The tilde is a shorthand notation for the discrete-time shift, i.e. for $q_{i}(n)=q_{i}$ we write $q_{i}(n+1)=\widetilde{q}_{i}$, and $q_{i}(n-1)={\underset{\sim}{i}}_{i}$. The variable $\kappa$ is the additional spectral parameter, whereas $\lambda$ is a parameter of the system. The matrices $e_{i j}$ are the standard elementary matrices whose entries are given by $\left(e_{i j}\right)_{k \ell}=\delta_{i, k} \delta_{j, \ell}$. The function $\Phi_{\kappa}$ is defined as

$$
\Phi_{\kappa}(x) \equiv \frac{\sigma(x+\kappa)}{\sigma(x) \sigma(\kappa)}
$$


where $\sigma(x)$ is the Weierstrass sigma-function, given by

$$
\sigma(x)=x \prod_{k, \ell \neq 0}\left(1-\frac{x}{\omega_{k \ell}}\right) \exp \left[\frac{x}{\omega_{k \ell}}+\frac{1}{2}\left(\frac{x}{\omega_{k \ell}}\right)^{2}\right],
$$

with $\omega_{k l}=2 k \omega_{1}+2 \ell \omega_{2}$ and $2 \omega_{1,2}$ being a fixed pair of the primitive periods, see e.g. [20]. The relations between the Weierstrass elliptic functions are given by

$$
\zeta(x)=\frac{\sigma^{\prime}(x)}{\sigma(x)}, \wp(x)=-\zeta^{\prime}(x),
$$

where $\sigma(x)$ and $\zeta(x)$ are odd functions and $\wp(x)$ is an even function of its argument. We recall also that the $\sigma(x)$ is an entire function, and $\zeta(x)$ is a meromorphic function having simple poles at $\omega_{k l}$, both being quasi-periodic, obeying

$$
\zeta\left(x+2 \omega_{1,2}\right)=\zeta(x)+2 \eta_{1,2} \quad, \quad \sigma\left(x+2 \omega_{1,2}\right)=-\sigma(x) e^{2 \eta_{1,2}\left(x+\omega_{1,2}\right)},
$$

in which $\eta_{1,2}$ satisfy $\eta_{1} \omega_{2}-\eta_{2} \omega_{1}=\frac{\pi i}{2}$, whereas $\wp(x)$ is periodic. From an algebraic point of view, the most important property of these elliptic functions is the existence of a number of functional relations, the most fundamental being

$$
\zeta(\alpha)+\zeta(\beta)+\zeta(\gamma)-\zeta(\alpha+\beta+\gamma)=\frac{\sigma(\alpha+\beta) \sigma(\beta+\gamma) \sigma(\gamma+\alpha)}{\sigma(\alpha) \sigma(\beta) \sigma(\gamma) \sigma(\alpha+\beta+\gamma)}
$$

From this relation, one can basically derive all important identities for the Weierstrass elliptic functions. For our purpose it can be recast into the form

$$
\Phi_{\kappa}(x) \Phi_{\kappa}(y)=\Phi_{\kappa}(x+y)[\zeta(\kappa)+\zeta(x)+\zeta(y)-\zeta(\kappa+x+y)] .
$$

The Ansatz for the Lax pair (2.1) is a natural one, in view of the fact that the matrix $L_{\kappa}$ corresponds to the Lax matrix of the continuum Ruijsenaars-Schneider (RS) model, cf. [13], whereas the matrix $M_{\kappa}$ is a natural choice by comparison with the earlier results obtained in [2], cf. also [3], for the discrete elliptic CM model.

Let us now consider the compatibility of the system (2.1). Assuming the Lax equation

$$
\widetilde{L}_{\kappa} M_{\kappa}=M_{\kappa} L_{\kappa}
$$

which implies the isospectrality of the discrete flow of the Lax matrix $L_{\kappa}$, we have from (2.6) that

$$
\begin{aligned}
& \sum_{\ell} \widetilde{h}_{\ell}^{2}\left[\zeta(\kappa)+\zeta\left(\widetilde{q}_{i}-\widetilde{q}_{\ell}+\lambda\right)+\zeta\left(\widetilde{q}_{\ell}-q_{j}+\lambda\right)-\zeta\left(\kappa+2 \lambda+\widetilde{q}_{i}-q_{j}\right)\right] \\
& =\sum_{\ell} h_{\ell}^{2}\left[\zeta(\kappa)+\zeta\left(\widetilde{q}_{i}-q_{\ell}+\lambda\right)+\zeta\left(q_{\ell}-q_{j}+\lambda\right)-\zeta\left(\kappa+2 \lambda+\widetilde{q}_{i}-q_{j}\right)\right] .
\end{aligned}
$$


Noting that the conservation law $\operatorname{tr} \widetilde{L}=\operatorname{tr} L$ implies:

$$
\sum_{\ell} \tilde{h}_{\ell}^{2}=\sum_{\ell} h_{\ell}^{2}
$$

we have the identity

$$
\sum_{\ell}\left[\widetilde{h}_{\ell}^{2} \zeta\left(\widetilde{q}_{i}-\widetilde{q}_{\ell}+\lambda\right)-h_{\ell}^{2} \zeta\left(\widetilde{q}_{i}-q_{\ell}+\lambda\right)\right]=-\sum_{\ell}\left[\widetilde{h}_{\ell}^{2} \zeta\left(\widetilde{q}_{\ell}-q_{j}+\lambda\right)-h_{\ell}^{2} \zeta\left(q_{\ell}-q_{j}+\lambda\right)\right]
$$

for all $i, j=1, \ldots, N$ : consequently, both sides of (2.9) must be independent of the (external) particle label. Thus, we find a coupled system of equations in terms of the variables $h_{i}, q_{i}$ of the form

$$
\begin{aligned}
& \sum_{\ell}\left[\widetilde{h}_{\ell}^{2} \zeta\left(q_{i}-\widetilde{q}_{\ell}-\lambda\right)-h_{\ell}^{2} \zeta\left(q_{i}-q_{\ell}-\lambda\right)\right]=-p, \\
& \sum_{\ell}\left[{\underset{\sim}{2}}_{\ell}^{2} \zeta\left(q_{i}-{\underset{\sim}{q}}_{\ell}+\lambda\right)-h_{\ell}^{2} \zeta\left(q_{i}-q_{\ell}+\lambda\right)\right]=\underset{\sim}{p},
\end{aligned}
$$

where $p$ does not carry a particle label. In principle $p$ can still depend on the discrete time-variable, but we will mostly consider it to be constant: a different choice will be taken only in Section 6. In that case, by eliminating the variables $h_{i}$ from (2.9), we get a closed set of equations in terms of the $q_{i}$.

In order to derive this closed set of equations for the variables $q_{i}$ we will make use of an elliptic version of the Lagrange interpolation formula, that was derived in [2]. In fact, we have the following statement:

Lemma: Consider $2 N$ noncoinciding complex numbers $x_{l}, y_{l}(l=1, \ldots, N)$. Then, the following formula holds true:

$$
\begin{array}{r}
\prod_{l=1}^{N} \frac{\sigma\left(\xi-x_{l}\right)}{\sigma\left(\xi-y_{l}\right)}=\sum_{l=1}^{N}\left[\zeta\left(\xi-y_{l}\right)-\zeta\left(x-y_{l}\right)\right] \frac{\prod_{k=1}^{N} \sigma\left(y_{l}-x_{k}\right)}{\prod_{\substack{k=1 \\
k \neq l}}^{N} \sigma\left(y_{l}-y_{k}\right)}, \\
\text { where } \sum_{l=1}^{N}\left(y_{l}-x_{l}\right)=0,
\end{array}
$$

and where $x$ stands for any one of the zeroes $x_{l}$ of the function on the left-hand side.

Eq. (2.11) can be derived from an elliptic version of the Cauchy identity for the determinants of the matrix $\left(\Phi_{\kappa}\left(x_{i}-y_{j}\right)\right)$. This Cauchy identity was proven and used in 
[12] to establish the commutativity of the quantum integrals for the continuum RS model. Actually, this identity goes back to Frobenius, [21], and can be proven also by purely combinatoric means starting from the fundamental identity (2.5). A statement similar to the one in the lemma was formulated in [22].

What is essential in (2.11) is the independence of the choice of $x$, which can be easily demonstrated by using the identity

$$
\sum_{i=1}^{N} \frac{\prod_{\substack{j=1 \\ j=1}}^{N} \sigma\left(y_{i}-x_{j}\right)}{\prod_{\substack{j=1 \\ j \neq i}}^{N} \sigma\left(y_{i}-y_{j}\right)}=0, \quad \sum_{i=1}^{N}\left(y_{i}-x_{i}\right)=0,
$$

which can be found e.g. in [23], p. 451.

Using now eq. (2.11) and applying the lemma to the elliptic fractional "polynomial"

$$
\prod_{k=1}^{N} \frac{\sigma\left(\xi-q_{k}+\lambda\right) \sigma\left(\xi-\widetilde{q}_{k}-\lambda\right)}{\sigma\left(\xi-q_{k}\right) \sigma\left(\xi-\widetilde{q}_{k}\right)}
$$

and taking $\xi=q_{j}-\lambda$, respectively $x=\widetilde{q}_{i}+\lambda$, we can by comparing with eq. (2.9) make the identifications

$$
\begin{gathered}
h_{\ell}^{2}=-p \frac{\prod_{k=1}^{N} \sigma\left(q_{\ell}-q_{k}+\lambda\right) \sigma\left(q_{\ell}-\widetilde{q}_{k}-\lambda\right)}{\left[\prod_{k \neq \ell} \sigma\left(q_{\ell}-q_{k}\right)\right] \prod_{k=1}^{N} \sigma\left(q_{\ell}-\widetilde{q}_{k}\right)}, \\
\widetilde{h}_{\ell}^{2}=p \frac{\prod_{k=1}^{N} \sigma\left(\widetilde{q}_{\ell}-q_{k}+\lambda\right) \sigma\left(\widetilde{q}_{\ell}-\widetilde{q}_{k}-\lambda\right)}{\left[\prod_{k \neq \ell} \sigma\left(\widetilde{q}_{\ell}-\widetilde{q}_{k}\right)\right] \prod_{k=1}^{N} \sigma\left(\widetilde{q}_{\ell}-q_{k}\right)}, \\
\ell=1, \ldots, N
\end{gathered}
$$

from which we obtain the following system of equations

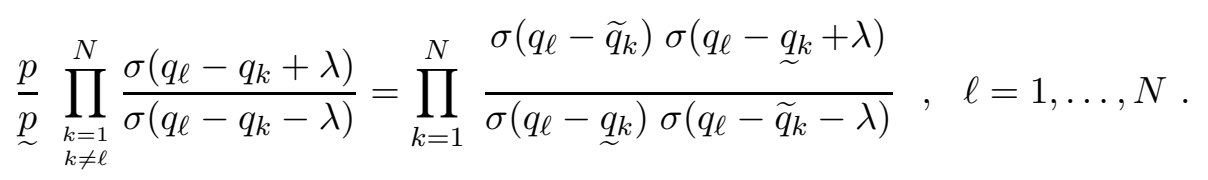

Eqs. (2.14) can be considered to be a product version of (2.9), and is a system of $N$ equations for $N+1$ unknowns, $q_{1}, \ldots, q_{N}$ and $p$. There is no equation for $p$ separately, and thus it should be a priori given in order to get a closed set of equations. The most natural choice is the one for which $p$ is constant, i.e. independent of the discrete timevariable. For convenience we will take it equal to the fixed value $p=-\sigma(\lambda)^{-1}$, but if we are interested in a continuum limit from $(2.10)$ we should take it of the order of the reciprocal of the discrete-time step. As already mentioned, a dynamical choice of $p$ arises 
at the end of Section 6 from the application of (2.14) to the Bethe Ansatz equations for the XYZ Heisenberg chain.

Thus, taking $p / \underset{\sim}{p}$ to be equal to unity in (2.14), we obtain the equations of motion of what we would like to call the discrete Ruijsenaars-Schneider model. It is given by a coupled set of algebraic equations, which, in fact, resemble closely the Bethe Ansatz equations (BAE's) for certain integrable quantum chains with impurities. We will make this connection more precise in Section 6. Here, we look at eqs. 2.14 as defining a discrete dynamical system, which amounts to an integrable symplectic correspondence, i.e. a multi-valued map in the sense of ref. [8].

Before proving the symplecticity and integrability of the correspondence, let us show first that the interpretation of (2.14) as a discrete version of the RS model is justified, by showing that an appropriate continuum limit will give us back the equations (1.1) of the continuum model. In fact, taking

$$
\begin{aligned}
& \widetilde{q}_{k} \mapsto q_{k}-\lambda+\epsilon \dot{q}_{k}+\frac{1}{2} \epsilon^{2} \ddot{q}_{k}+\ldots, \\
& q_{k} \mapsto q_{k}+\lambda-\epsilon \dot{q}_{k}+\frac{1}{2} \epsilon^{2} \ddot{q}_{k}+\ldots,
\end{aligned}
$$

and developing with respect to the small parameter $\epsilon$, we obtain for the leading term

$$
\frac{\ddot{q}_{\ell}}{\dot{q}_{\ell}}=\sum_{k \neq \ell} \dot{q}_{k}\left[2 \zeta\left(q_{\ell}-q_{k}\right)-\zeta\left(q_{\ell}-q_{k}+\lambda\right)-\zeta\left(q_{\ell}-q_{k}-\lambda\right)\right],
$$

from which we recover eqs. (1.1) using the relation

$$
\zeta(x+\lambda)-\zeta(x)-\zeta(\lambda)=\frac{1}{2} \frac{\wp^{\prime}(\lambda)-\wp^{\prime}(x)}{\wp(\lambda)-\wp(x)} .
$$

Let us finally remark that the non-relativistic limit is obtained by letting the parameter $\lambda$ go to zero. In fact, in this limit the variables $h_{i}^{2}$ will behave as

$$
h_{i}^{2} \mapsto 1+\lambda\left(\sum_{k \neq \ell} \zeta\left(q_{\ell}-q_{k}\right)-\sum_{k=1}^{N} \zeta\left(q_{\ell}-\widetilde{q}_{k}\right)\right)+\mathcal{O}\left(\lambda^{2}\right) .
$$

As $\lambda \rightarrow 0$, we will obtain the matrix $M_{\kappa}$ of the non-relativistic case immediately, whereas for $L_{\kappa}$ we thus find

$$
L_{\kappa} \mapsto \lambda^{-1}+\sum_{i} p_{i} e_{i i}+\sum_{i \neq j} \Phi_{\kappa}\left(q_{i}-q_{j}\right) e_{i j}+\mathcal{O}(\lambda)
$$


where the $p_{i}$ are the expressions between brackets on the right-hand side of the arrow in (2.17). Thus, we recover the Lax representation of the non-relativistic case as given in [2].

\section{Symplectic Structure and Integrability}

In order to demonstrate the integrability of the discrete-time $\mathrm{RS}$ model, given by the equations of motion (2.14 together with $p=\underset{\sim}{p}$, we need to establish an invariant symplectic structure. Following the philosophy of previous papers, (cf. e.g. 24 and references therein), this can be assessed on the basis of an action principle, cf. also [8, 19].

It is easy to note that an action for the discrete equations (2.14) is given by

$$
S=\sum_{n} \mathcal{L}(q(n), q(n+1))
$$

in which

$$
\mathcal{L}(q, \widetilde{q})=\sum_{k, \ell=1}^{N}\left[f\left(q_{\ell}-\widetilde{q}_{k}\right)-f\left(q_{\ell}-\widetilde{q}_{k}-\lambda\right)\right]-\sum_{\substack{k, \ell=1 \\ k \neq \ell}}^{N} f\left(q_{\ell}-q_{k}+\lambda\right),
$$

with the function $f(x)$ given by

$$
f(x)=\int^{x} \log |\sigma(\xi)| d \xi
$$

Let us point out that the function $f$ appearing here can be considered to be an elliptic version of the Euler dilogarithm function. The discrete Euler-Lagrange equations

$$
\frac{\partial \mathcal{L}}{\partial \widetilde{q}_{\ell}}+\frac{\widetilde{\partial \mathcal{L}}}{\partial q_{\ell}}=0 \quad, \quad \ell=1, \ldots, N
$$

are easily seen to lead to eq. (2.14). The canonical momenta $p_{\ell}$ are found from

$$
\widetilde{p}_{\ell}=\frac{\partial \mathcal{L}}{\partial \widetilde{q}_{\ell}}=\sum_{k=1}^{N}\left(-\log \left|\sigma\left(\widetilde{q}_{\ell}-q_{k}\right)\right|+\log \left|\sigma\left(\widetilde{q}_{\ell}-q_{k}+\lambda\right)\right|\right) .
$$

As a consequence we have that the symplectic form $\Omega=\sum_{k} d p_{k} \wedge d q_{k}$ is invariant under the correspondence, which implies that any branch of the correspondence defines a canonical transformation with respect to the standard Poisson brackets given by

$$
\left\{p_{k}, q_{\ell}\right\}=\delta_{k, \ell}, \quad\left\{p_{k}, p_{\ell}\right\}=\left\{q_{k}, q_{\ell}\right\}=0
$$


We will now show that in terms of the canonical variables, the Lax matrix $L_{\kappa}$ in (2.1) has the same form as the one of the continuum RS model. In fact, expressing the variables $h_{\ell}^{2}$ in terms of the canonical variables, we obtain

$$
h_{\ell}^{2}=e^{p_{\ell}} \prod_{k \neq \ell} \frac{\sigma\left(q_{\ell}-q_{k}-\lambda\right)}{\sigma\left(q_{\ell}-q_{k}\right)},
$$

which we will refer to as the Ruijsenaars variables, [10]. In terms of these variables we have the Poisson brackets

$$
\begin{aligned}
& \left\{q_{k}, q_{\ell}\right\}=0, \quad\left\{\log h_{k}^{2}, q_{\ell}\right\}=\delta_{k, \ell}, \\
& \left\{\log h_{k}^{2}, \log h_{\ell}^{2}\right\}=\zeta\left(q_{k}-q_{\ell}+\lambda\right)+\zeta\left(q_{k}-q_{\ell}-\lambda\right)-2 \zeta\left(q_{k}-q_{\ell}\right), \quad k \neq \ell .
\end{aligned}
$$

Now, there remains not so much work left to establish the integrability. In fact, having expressed the Lax matrix $L_{\kappa}$ in terms of the original variables, and having verified that

it takes the same form as in the continuous case, we can rely on the proof of involutivity that was produced by Ruijsenaars (in Appendix A of [10]) to assess the involutivity of the invariants of the discrete model as well. Naturally, the invariants of the correspondence, given by

$$
I_{k}=\operatorname{tr} L^{k}=\widetilde{I}_{k} \quad, \quad k=1, \ldots, N,
$$

as functions of the canonical variables are the same as in the continuum model.

Thus, we obtain the statement of involutivity of the invariants, i.e.,

$$
\left\{I_{k}, I_{\ell}\right\}=\left\{\operatorname{Tr}\left(L^{k}\right), \operatorname{Tr}\left(L^{\ell}\right)\right\}=0 \quad \text { for all } k, \ell=1,2, \ldots
$$

It is a theorem by Veselov that the involutivity of the invariants lead to the linearization of the discrete flow on tori, similar to the continuous-time situation, cf. [8]. (For a simple argument see also [19]).

\section{Exact Solutions}

Let us now consider exact solutions of the discrete RS model in two special cases: i) the rational limit, ii) the hyperbolic (trigonometric) limit. Here, again, we restrict ourselves to the case $p=\underset{\sim}{p}$, which will then lead to an explicit integration scheme from the Lax 
pair, as we shall demonstrate below. If, more generally, we do not fix $p$, the initial value problem becomes implicit, having to take into account initial values for the $p$ also. We shall not pursue this latter case in this paper.

The main line of reasoning in both limiting cases is very similar to the one followed in [1, 2] for the non-relativistic discrete CM model. In the discrete situation the initial value problem is posed by asking to find $\left\{q_{i}(n)\right\}$ for given initial data $\left\{q_{i}(0)\right\}$ and $\left\{q_{i}(1) \equiv \widetilde{q}_{i}(0)\right\}$, where $q_{i}(n)$ denotes the position of the particles $q_{i}$ at the $n$th time-step. The solution to this problem is obtained for both the rational as well as the hyperbolic (trigonometric) limits by solving a secular problem, namely by determining the eigenvalues of an $N \times N$ matrix which can be explicitely calculated from the initial data for any discrete-time value $n$. However, one may ask the legitimate question what one has gained from this, as one has reduced the problem of solving one set of algebraic equations (given by the discrete equations of motion (2.14) ) to another problem of solving an algebraic equation (namely the factorization of a characteristic determinant). In the first case, nonetheless, we have to solve the system step by step at each iteration of the map, whence the issue of the complexity of the solution (i.e. the growth of the number of iterates) becomes imminent. In view of the integrability of the correspondence, one can at most have only polynomial growth, as Veselov has proven in [25], and this is immediately clear from the exact solution, because the only growth comes from the permutations of eigenvalues. In fact, there are $N$ ! permutations at each iteration of the map, but whatever be the permutation chosen at a certain step, it will end into one of the $N$ ! possible permutations at the following step: the branches will "cross" each other with no divergence.

\section{i) Rational Case}

This is the limit that both periods tend to infinity, i.e. $2 \omega_{1} \rightarrow \infty, 2 \omega_{2} \rightarrow i \infty$, in which case we can make the substitutions

$$
\sigma(x) \rightarrow x \quad, \quad \zeta(x) \rightarrow \frac{1}{x} \quad, \quad \wp(x) \rightarrow \frac{1}{x^{2}} .
$$

Then, the Lax matrices take the form

$$
L_{\kappa}=\frac{1}{\kappa} h^{t} h+L_{0} \quad, \quad M_{\kappa}=\frac{1}{\kappa} \widetilde{h}^{t} h+M_{0}
$$


where $h$ denotes the (column-)vector with entries $h_{i}$, the (row-)vector ${ }^{t} h$ being its transposed, and in which

$$
L_{0}=\sum_{i, j=1}^{N} \frac{h_{i} h_{j}}{\lambda+q_{i}-q_{j}} e_{i j} \quad, \quad M_{0}=\sum_{i, j=1}^{N} \frac{\widetilde{h}_{i} h_{j}}{\lambda+\widetilde{q}_{i}-q_{j}} e_{i j} .
$$

From the form of the Lax matrices (4.2), we can then derive the following relations

$$
\begin{aligned}
\lambda M_{0}+\widetilde{Q} M_{0}-M_{0} Q & =\widetilde{h}^{t} h, \\
\lambda L_{0}+Q L_{0}-L_{0} Q & =h^{t} h,
\end{aligned}
$$

where we have set

$$
Q=\sum_{i=1}^{N} q_{i} e_{i i}
$$

On the other hand, from the Lax equation (2.7) and inserting (4.1), we obtain the relations

$$
\begin{aligned}
\widetilde{L}_{0} M_{0} & =M_{0} L_{0}, \\
\widetilde{L}_{0} \widetilde{h}-M_{0} h & =-p \widetilde{h}, \\
{ }^{t} h L_{0}-{ }^{t} \widetilde{h} M_{0} & =-p^{t} h,
\end{aligned}
$$

together with the conservation law ${ }^{t h \cdot h}={ }^{t} h \cdot h$. Consequently, we can put

$$
M_{0}=\widetilde{U}_{0} U_{0}^{-1} \quad, \quad L_{0}=U_{0} \Lambda U_{0}^{-1},
$$

where $U_{0}$ is an invertible $N \times N$ matrix, and where the matrix $\Lambda$ is constant, $\widetilde{\Lambda}=\Lambda$, as a consequence of (4.5a). We assume that $\Lambda$ can be chosen to be a diagonal matrix, but this is not essential for what follows. Then, introducing

$$
V \equiv U_{0}^{-1} Q U_{0} \quad, \quad r \equiv U_{0}^{-1} \cdot h \quad, \quad{ }^{t} s \equiv{ }^{t} h \cdot U_{0}
$$

we obtain from $(4.5)$ and $(4.6)$

$$
(p I+\Lambda) \cdot \widetilde{r}=r \quad, \quad{ }^{t} s \cdot(p I+\Lambda)={ }^{t} \widetilde{s},
$$

in which $I$ denotes the $N \times N$ unit matrix, as well as from 4.3a and 4.3b

$$
\lambda+\widetilde{V}-V=\widetilde{r}^{t} s, \lambda \Lambda+[V, \Lambda]=r^{t} s
$$


together with the conservation law $\widetilde{r^{t}} s=r^{t} s$. Eliminating the dyadic $r^{t} s$ from eq. (4.9) by making use of (4.8), we find the linear equation

$$
\widetilde{V}=(p I+\Lambda)^{-1} V(p I+\Lambda)-\frac{p \lambda}{p I+\Lambda},
$$

which can be immediately solved to give

$$
V(n)=(p I+\Lambda)^{-n}\left[V(0)-n \frac{p \lambda}{p I+\Lambda}\right](\Lambda+p I)^{n} .
$$

Thus, the solution of the discrete RS model in the rational case is given by the following statement : the position coordinates $\left\{q_{i}(n)\right\}$ of the particles at the discrete time $n$, evolving under the discrete equations of motion (2.14) (in the rational limit $\sigma(x) \mapsto x$ ), are given by the eigenvalues of the matrix:

$$
Q(0)-n p \lambda\left(p I+L_{0}(0)\right)^{-1}
$$

where $p$ can be chosen equal to unity without loss of generality.

The choice of $p$ only affects a scaling of the variables $h_{i}$ in (2.1), which enter the initial value of the matrix $L_{0}(0)$.

\section{ii) Hyperbolic (Trigonometric) Case}

In the hyperbolic limit $2 \omega_{1} \rightarrow \infty, 2 \omega_{2}=\frac{1}{2} \pi i$, in which case we can make the substitutions

$$
\sigma(x) \rightarrow \sinh (x) \quad, \quad \zeta(x) \rightarrow \operatorname{coth}(x) \quad, \quad \wp(x) \rightarrow \sinh ^{-2}(x)
$$

(after an appropriate gauge transformation of the Lax matrix with a diagonal matrix), leading to

$$
\Phi_{\kappa}(x) \rightarrow \operatorname{coth}(\kappa)+\operatorname{coth}(x) .
$$

In this case we obtain for the Lax matrices (2.1)

$$
L_{\kappa}=L_{0}+(\operatorname{coth} \kappa+\gamma) h^{t} h, \quad M_{\kappa}=M_{0}+(\operatorname{coth} \kappa+\gamma) \widetilde{h}^{t} h,
$$

where $\gamma$ can be chosen at our convenience. In this case the reduced Lax matrices $L_{0}$ and $M_{0}$ are given by

$$
\begin{aligned}
L_{0} & =\sum_{i, j=1}^{N} h_{i} h_{j}\left(\operatorname{coth}\left(q_{i}-q_{j}+\lambda\right)-\gamma\right) e_{i j}, \\
M_{0} & =\sum_{i, j=1}^{N} \widetilde{h}_{i} h_{j}\left(\operatorname{coth}\left(\widetilde{q}_{i}-q_{j}+\lambda\right)-\gamma\right) e_{i j} .
\end{aligned}
$$


For them, as a consequence of the 'splitting off' of the terms with the spectral parameter $\operatorname{coth} \kappa$, we get again the system of equations (4.5). From eqs. (4.15) we derive that

$$
\begin{aligned}
& e^{2 \lambda} e^{2 Q}\left(L_{0}+\gamma h^{t} h\right)-\left(L_{0}+\gamma h^{t} h\right) e^{2 Q}=e^{2 \lambda} e^{2 Q} h^{t} h+h^{t} h e^{2 Q}, \\
& e^{2 \lambda} e^{2 \widetilde{Q}}\left(M_{0}+\gamma \widetilde{h}^{t} h\right)-\left(M_{0}+\gamma \widetilde{h}^{t} h\right) e^{2 Q}=e^{2 \lambda} e^{2 \widetilde{Q}} \widetilde{h}^{t} h+\widetilde{h}^{t} h e^{2 Q},
\end{aligned}
$$

in which $Q, h$ and ${ }^{t} h$ are given as before. We again make the identifications (4.6), and introduce $V, r, s$ as in (4.7), for which we subsequently derive the relations

$$
\begin{aligned}
e^{2 \lambda} e^{2 V}\left(\Lambda+(\gamma-1) r^{t} s\right) & =\left(\Lambda+(\gamma+1) r^{t} s\right) e^{2 V} \\
e^{2 \lambda} e^{2 \widetilde{V}}\left(I+(\gamma-1) \widetilde{r}^{t} s\right) & =\left(I+(\gamma+1) \widetilde{r}^{t} s\right) e^{2 V}
\end{aligned}
$$

Choosing now $\gamma=1$, and using (4.8) we can again eliminate the dyadic $r^{t} s$ and explicitely solve for $\exp (2 V)$. We obtain

$$
e^{2 \widetilde{V}}=(p+\Lambda)^{-1} e^{2 V}\left(p e^{-2 \lambda}+\Lambda\right)
$$

which can be immediately integrated to yield

$$
e^{2 V(n)}=(\Lambda+p I)^{-n} e^{2 V(0)}\left(\Lambda+p e^{-2 \lambda} I\right)^{n},
$$

the $V(0)$ and $U(0)$ as well as $\Lambda$ being determined from the initial data $Q(0)$ and $Q(1)$. Thus, rewriting (4.19) we obtain the following result: the exponentials of the position coordinates $\left\{e^{2 q_{i}(n)}\right\}$ of the particles at the discrete time $n$, evolving under the discrete equations of motion (2.14) (in the hyperbolic limit $\sigma(x) \mapsto \sinh (x)$ ), are given by the eigenvalues of the matrix:

$$
\left(L_{0}(0)+p I\right)^{-n} e^{2 Q(0)}\left(L_{0}(0)+p e^{-2 \lambda} I\right)^{n},
$$

where $p$ can be chosen equal to unity without loss of generality.

The choice of $p$ only affects a scaling of the variables $h_{i}$ in (2.1), which can be accounted for in the calculation of the $h_{i}(0)$ from eq. (2.13). We note again that at each discrete-time value the positions of the particles is uniquely determined up to a permutation.

We finally remark that the trigonometric limit of the elliptic functions $2 \omega_{1}=\frac{1}{2} \pi$, $2 \omega_{2} \rightarrow i \infty$ is integrated along similar lines after doing the replacements

$$
\sigma(x) \rightarrow \sin x \quad, \quad \zeta(x) \rightarrow \cot x
$$

We shall omit the details. 


\section{Connection with Discrete Soliton Systems}

In [1, 3], the discrete CM model was obtained from pole-solutions of a lattice version of the Kadomtsev-Petviashvili (KP) equation. The idea that integrable particle models of CM type are connected with soliton equations goes back already to the late seventies, cf. [26] and also [27, 28], but had never been applied to discrete soliton equations. In [10], but maybe a bit more transparently in [29], a connection between the relativistic particle model and soliton solutions of nonlinear integrable PDE's was established. It is a natural question to ask whether such a connection also exists on the discrete level.

In order to establish such a connection, let us investigate more closely the trigonometric solution of the previous section. After a gauge transformation with a diagonal matrix of the form $P=\left[(\Lambda+p I)\left(\Lambda+e^{-2 \lambda} p I\right)\right]^{1 / 2}$, we can transform $e^{2 V}=P^{-n} W P^{n}$ into

$$
W_{i j}=-2 \frac{\rho_{i} \rho_{j}}{\lambda_{i}-\omega \lambda_{j}}
$$

in which $\rho_{i} \equiv\left(P^{n} \cdot r\right)_{i}=\left({ }^{t} s \cdot e^{2 V} P^{-n}\right)_{i}$, and $\omega \equiv e^{2 \lambda}$. The dependence on the discrete (time-)variable is given by

$$
\widetilde{\rho}_{i}=\left(\frac{\lambda_{i}+\omega^{-1} p}{\lambda_{i}+p}\right)^{1 / 2} \rho_{i},
$$

leading to the equations for $W$

$$
\begin{aligned}
& \Lambda W-\omega W \Lambda=-2 \rho^{t} \rho, \\
& P W-\omega \widetilde{W} P=-2 \widetilde{\rho}^{t} \rho,
\end{aligned}
$$

We can then introduce the characteristic polynomial

$$
\tau(\xi)=\prod_{j=1}^{N}\left(\xi+e^{2 q_{j}}\right)=\operatorname{det}(\xi I+W),
$$

and show that $\tau(\xi)$ plays the role of the tau-function of a discrete soliton system. In fact, $W$ is the kernel of the integral operator in the soliton sector that stands at the basis of the so-called direct linearization approach, cf. e.g. 30]-32].

To derive the relevant equations directly from the resolvent of the matrix $W$, we proceed as follows. First, using eq. (5.3b), we can perform the following simple calculation

$$
\frac{\widetilde{\tau}(\xi)}{\tau(\omega \xi)}=\operatorname{det}\left((\omega \xi I+W)^{-1}(\xi I+\widetilde{W})\right)
$$




$$
\begin{aligned}
& =\operatorname{det}\left[(\omega \xi I+W)^{-1}\left(\xi I+\omega^{-1} W+2 \omega^{-1}(\Lambda+p I)^{-1} \cdot \rho^{t} \rho\right)\right] \\
& =\omega^{-N} \operatorname{det}\left(I+(\omega \xi I+W)^{-1}(\Lambda+p I)^{-1} \cdot \rho^{t} \rho\right),
\end{aligned}
$$

leading to

$$
v(\omega \xi) \equiv 1+{ }^{t} \rho \cdot(\omega \xi I+W)^{-1}(\Lambda+p I)^{-1} \cdot \rho=\omega^{N} \frac{\widetilde{\tau}(\xi)}{\tau(\omega \xi)} .
$$

which will turn out to be one of the variables governed by the soliton system. In order to derive discrete soliton equations for $\tau(\xi)$ or $v$, we introduce the $N$-component vectors

$$
u_{j}(\xi) \equiv(\xi I+W)^{-1} \Lambda^{j} \cdot \rho,{ }^{t} u_{j}(\xi) \equiv{ }^{t} \rho \cdot \Lambda^{j}(\xi I+W)^{-1},
$$

as well as the scalar variables

$$
U_{i j}(\xi) \equiv 2^{t} \rho \cdot \Lambda^{j}(\xi I+W)^{-1} \Lambda^{i} \cdot \rho
$$

$(i, j \in \mathbf{Z})$. Making use of the relations (5.3), we can derive the following set of recursive relations between the different vectors $u_{j}(\xi)$ and ${ }^{t} u_{j}(\xi)$

$$
\begin{aligned}
\omega P \cdot u_{j}(\xi) & =p \widetilde{u}_{j}\left(\omega^{-1} \xi\right)+\widetilde{u}_{j+1}\left(\omega^{-1} \xi\right)+U_{j, 0}(\xi) \widetilde{u}_{0}\left(\omega^{-1} \xi\right) \\
{ }^{t} \widetilde{u}_{j}(\xi) \cdot P & =\omega^{t} u_{j+1}(\omega \xi)+p^{t} u_{j}(\omega \xi)-{ }^{t} u_{0}(\omega \xi) \widetilde{U}_{0, j}(\xi) .
\end{aligned}
$$

Using the definition $(5.6 \mathrm{~b})$ as well as $(5.2)$, we can derive also

$$
p \widetilde{U}_{i j}(\xi)+\widetilde{U}_{i+1, j}(\xi)=p U_{i j}(\omega \xi)+\omega U_{i, j+1}(\omega \xi)-U_{i, 0}(\omega \xi) \widetilde{U}_{0, j}(\xi)
$$

At this point we need to reflect a moment on the role of the discrete-time shift. The shift $U_{i j}(\xi) \mapsto \widetilde{U}_{i j}(\xi)$ is 'labeled' by the variable $p$, which can be identified as the (reciprocal) of the lattice parameter. Let us assume that the variables $U_{i j}(\xi)$ do not depend on only one discrete time-variable $n$, but on a number of them, say $n_{\alpha},(\alpha=1,2,3, \ldots)$, and that the corresponding discrete flows are compatible, each associated with its own parameter $p$, i.e. $p_{1}, p_{2}, p_{3}, \ldots$ This means that $U$ is a function of these independent time-variables, i.e. $U_{i j}(\xi)=U_{i j}\left(\xi ; n_{1}, n_{2}, \ldots\right)$. In that case we have for instance that $\widehat{\widetilde{U}}_{i j}\left(\xi ; n_{1}, n_{2}, \ldots\right)=$ $U_{i j}\left(\xi ; n_{1}+1, n_{2}+1, \ldots\right)=\widetilde{\widehat{U}}_{i j}\left(\xi ; n_{1}, n_{2}, \ldots\right)$, etc. , the - corresponding to the translation in the second discrete variable. Of course, this puts extra compatibility conditions on the eigenvalues $e^{2 q_{j}}$ of the kernel $W$ given in (5.1) of the soliton solutions. In fact, the particle-coordinates $q_{j}$ can be shown to obey some two-dimensional lattice equations in 
terms of the shifts $\sim$ and ${ }^{\wedge}$, and the compatibility of such equations were investigated in [2] for the non-relativistic situation. Here, we restrict ourselves to obtaining from (5.70) closed-form nonlinear partial difference equations for special elements or combinations of elements of $U_{i j}$, i.e. partial difference versions of the well-known soliton systems. These difference systems were investigated in detail in a number of earlier papers, (cf. [33] for a review, and references therein), on the basis of the system of equations (5.7a)-(5.7c). Thus here we will only present the results, referring to those papers for their derivation.

In fact, for the special element $u_{n_{1}, n_{2}, n_{3}} \equiv U_{0,0}\left(\omega^{-\left(n_{1}+n_{2}+n_{3}\right)} \xi ; n_{1}, n_{2}, n_{3}\right)$, we can derive the partial difference equation

$$
\left(p_{1}-p_{2}+u_{n_{1}+1, n_{2}, n_{3}}-u_{n_{1}, n_{2}+1, n_{3}}\right)\left(p_{3}+u_{n_{1}+1, n_{2}+1, n_{3}}\right)+\text { cycl. perm. }=0,
$$

which is a lattice version of the Kadomtsev-Petviashvili (KP) equation, cf. [30, 31]. In [2, 3] pole solutions of this equation were investigated associated with a discretization of the non-relativistic Calogero-Moser model. The relation to the $\tau$-function $\tau_{n_{1}, n_{2}, n_{3}} \equiv$ $\tau\left(\omega^{-\left(n_{1}+n_{2}+n_{3}\right)} \xi ; n_{1}, n_{2}, n_{3}\right)$ is given by

$$
p_{1}-p_{2}+u_{n_{1}, n_{2}+1, n_{3}}-u_{n_{1}+1, n_{2}, n_{3}}=\left(p_{1}-p_{2}\right) \frac{\tau_{n_{1}+1, n_{2}+1, n_{3}} \tau_{n_{1}, n_{2}, n_{3}}}{\tau_{n_{1}+1, n_{2}, n_{3}} \tau_{n_{1}, n_{2}+1, n_{3}}},
$$

from which one can derive the bilinear equation

$$
\left(p_{1}-p_{2}\right) \tau_{n_{1}+1, n_{2}+1, n_{3}}\left(\omega^{-1} \xi\right) \tau_{n_{1}, n_{2}, n_{3}+1}(\xi)+\text { cycl. perm. }=0
$$

which was first presented in [34], cf. also [35]. Eq. (5.10) is related also to the following version of the lattice (modified) KP equation, [35],

$$
\left(p_{1}-p_{2}\right)\left(\frac{v_{n_{1}, n_{2}+1, n_{3}+1}}{v_{n_{1}, n_{2}, n_{3}+1}}-\frac{v_{n_{1}+1, n_{2}+1, n_{3}}}{v_{n_{1}+1, n_{2}, n_{3}}}\right)=\left(p_{1}-p_{3}\right)\left(\frac{v_{n_{1}, n_{2}+1, n_{3}+1}}{v_{n_{1}, n_{2}+1, n_{3}}}-\frac{v_{n_{1}+1, n_{2}, n_{3}+1}}{v_{n_{1}+1, n_{2}, n_{3}}}\right),
$$

in terms of the variable $v_{n_{1}, n_{2}, n_{3}}=v\left(\omega^{-\left(n_{1}+n_{2}+n_{3}\right)} \xi ; n_{1}, n_{2}, n_{3}\right)$, with $v$ being defined in (5.5), (taking $\left.p_{1}=p\right)$.

Finally, we mention that special reductions of the above equations arising by imposing additional symmetries on the soliton solutions, will lead to the lattice soliton systems of Gel'fand-Dikii type that were introduced in [32]. In fact, taking the parameter $\omega$ equal to a root of unity, we can derive additional constraints on the system of equations given by 
(5.7a)-(5.7d). Thus, we find in the particular case of $\omega=-1$ for $u$ the following lattice version of the Korteweg-de Vries equation

$$
\left(p_{1}-p_{2}+u_{n_{1}, n_{2}+1}-u_{n_{1}+1, n_{2}}\right)\left(p_{1}+p_{2}+u_{n_{1}, n_{2}}-u_{n_{1}+1, n_{2}+1}\right)=p_{1}^{2}-p_{2}^{2},
$$

cf. [32], which is related to the following bilinear equation in terms of the $\tau$-function

$$
\left(p_{1}+p_{2}\right) \tau_{n_{1}-1, n_{2}+1} \tau_{n_{1}+1, n_{2}}+\left(p_{1}-p_{2}\right) \tau_{n_{1}-1, n_{2}} \tau_{n_{1}+1, n_{2}+1}=2 p_{1} \tau_{n_{1}, n_{2}} \tau_{n_{1}, n_{2}+1}
$$

It is this equation that corresponds in the continuum limit exactly to the special case considered in [29]. Similar equations can be derived for other values of $\omega$ when $|\omega|=1$, starting from the results presented in 32].

\section{Connection with Bethe Ansatz Equations}

We already remarked above that the equations of motion of the discrete RuijsenaarsSchneider model, (2.14), resemble closely the form of Bethe Ansatz equations (BAE's) for certain integrable quantum models. In this section we will make this connection more precise. Let us first focuss on the hyperbolic limit of eqs. (2.14), and connect it to the

BAE's for the XXZ spin- $\frac{1}{2}$ Heisenberg magnet, cf. e.g. 36-38 and references therein. After that, we will show that the general form of (2.14) in the elliptic case is connected to the generalized Bethe Ansatz (BA) for the Heisenberg XYZ model, proposed first in [39] and developed further in [40]. We will not treat the correspondence in the rational (or XXX) case since it can be obtained by a simple limit from the hyperbolic case.

Let us, thus, consider the quadratic $R$-matrix algebra

$$
R^{(12)}(u-v) T^{(1)}(u) T^{(2)}(v)=T^{(2)}(v) T^{(1)}(u) R^{(12)}(u-v)
$$

which is one of the central relations in the quantum inverse scattering method (QISM), (cf. [37] for an early, and [38, 41] for more recent reviews), and let us consider the $R$-matrix 
for the spin- $\frac{1}{2}$ XXZ Heisenberg magnet,

$$
R^{(12)}(u)=\left(\begin{array}{cccc}
a & 0 & 0 & 0 \\
0 & b & c & 0 \\
0 & c & b & 0 \\
0 & 0 & 0 & a
\end{array}\right),
$$

in which

$$
a=\sinh (u+\eta), \quad b=\sinh u, c=\sinh \eta
$$

The quantum $L$-operator on each site $k$ of the spin chain has the form

$$
L_{k}(u)=\left(\begin{array}{cc}
\sinh \left(u+\frac{1}{2} \eta \sigma_{k}^{3}\right) & \sinh (\eta) \sigma_{k}^{-} \\
\sinh (\eta) \sigma_{k}^{+} & \sinh \left(u-\frac{1}{2} \eta \sigma_{k}^{3}\right)
\end{array}\right),
$$

where $\sigma_{k}^{ \pm}=\frac{1}{2}\left(\sigma_{k}^{1} \pm i \sigma_{k}^{2}\right), \sigma_{k}^{1,2,3}$ being the Pauli matrices on site $k$, (i.e. $\sigma_{k}^{\alpha}=\mathbf{1} \otimes \ldots \otimes$ $\sigma^{\alpha} \otimes \ldots \otimes 1$ with $\sigma^{\alpha}$ on the $k$ th entry of the tensor product). Let us now construct the following monodromy matrix

$$
T(u)=L_{M}\left(u-\delta_{M}\right) \ldots L_{2}\left(u-\delta_{2}\right) L_{1}\left(u-\delta_{1}\right)=\left(\begin{array}{cc}
A(u) & B(u) \\
C(u) & D(u)
\end{array}\right),
$$

where $M$ is the length of the spin chain, and in which the $\delta_{k}, k=1, \ldots, M$ are impurity parameters. Both the $L_{k}(u)$ as well as $T(u)$ obey the relation (6.1). Let us now recall the standard algebraic Bethe Ansatz construction, [37]- 41], i.e. there is a vacuum state $\Omega_{M}$, which is an eigenvector of the diagonal entries $A(u), D(u)$ of the monodromy matrix, and which is annihilated by the operator $C(u)$

$$
A(u) \Omega_{M}=a(u) \Omega_{M} \quad, \quad D(u) \Omega_{M}=d(u) \Omega_{M}, C(u) \Omega_{M}=0 .
$$

As the monodromy matrix is a comultiplication of the $L$-operators along the sites of the chain, we have that

$$
a(u)=\prod_{k=1}^{M} a_{k}(u), \quad d(u)=\prod_{k=1}^{M} d_{k}(u),
$$

where the functions $a_{k}(u)$ and $d_{k}(u)$ are the eigenvalues of the vacuum for the diagonal entries of the corresponding $L$-operator on site $k$. The algebraic Bethe Ansatz amounts to the creation of an eigenstate of the form

$$
\Psi\left(q_{1}, \ldots, q_{N}\right)=\prod_{j=1}^{N} B\left(q_{j}\right) \Omega_{M}
$$


of the trace of the monodromy matrix $A(u)+D(u)$,

$$
(A(u)+D(u)) \Psi=t(u) \Psi,
$$

with $t(u)$ being the corresponding eigenvalue. We have then the following proposition:

Proposition: $\Psi$ is an eigenfunction of the trace of the monodromy matrix iff the numbers $q_{j}$ satisfy the Bethe Ansatz equations

$$
\prod_{\substack{k=1 \\ k \neq \ell}}^{N} \frac{\sinh \left(q_{\ell}-q_{k}+\eta\right)}{\sinh \left(q_{\ell}-q_{k}-\eta\right)}=\frac{a\left(q_{\ell}\right)}{d\left(q_{\ell}\right)}, \quad \ell=1, \ldots, N .
$$

In the case of the monodromy matrix (6.4), the functions $a(u), d(u)$ take the form

$$
a(u)=\prod_{k=1}^{M} \sinh \left(u-\delta_{k}+\frac{1}{2} \eta\right), \quad d(u)=\prod_{k=1}^{M} \sinh \left(u-\delta_{k}-\frac{1}{2} \eta\right),
$$

which in the special case that $M=2 N$ lead to the BAE's

$$
\prod_{\substack{k=1 \\ k \neq \ell}}^{N} \frac{\sinh \left(q_{\ell}-q_{k}+\lambda\right)}{\sinh \left(q_{\ell}-q_{k}-\lambda\right)}=\prod_{k=1}^{N} \frac{\sinh \left(q_{\ell}-\widetilde{q}_{k}\right) \sinh \left(q_{\ell}-q_{\sim}+\lambda\right)}{\sinh \left(q_{\ell}-{\underset{\sim}{q}}_{k}\right) \sinh \left(q_{\ell}-\widetilde{q}_{k}-\lambda\right)}, \quad \ell=1, \ldots, N,
$$

i.e. eq. (2.14) in the hyperbolic limit $(\sigma(x) \mapsto \sinh (x))$ with the identifications $\lambda=$ $\eta, \widetilde{q}_{k}=\delta_{k}-\frac{1}{2} \eta,{\underset{\sim}{q}}_{k}=\delta_{k+N}+\frac{1}{2} \eta,(k=1, \ldots, N)$. Thus, the discrete RS system in the hyperbolic limit can be reinterpreted as the BAE's for the spin- $\frac{1}{2}$ XXZ Heisenberg chain with the number of spins (impurities) equal to twice the excitation number of the eigenstate. We should stress that within such an identification the impurities play the role of the $N$ particle coordinates at times $n-1$ and $n+1(\underset{q}{\operatorname{resp}}$. $\widetilde{q})$, which means that the integrable correspondence mixes the parameters of the quantum model (i.e. the impurities) and the parameters of a solution given by the Bethe wavefunction.

To make the connection with the elliptic case we have to consider the QISM for the XYZ Heisenberg model that was treated in [39] for the spin- $\frac{1}{2}$ situation. For arbitrary spin one needs to consider the algebras introduced by Sklyanin in [42]. However, we are interested here in the inhomogeneous spin- $\frac{1}{2}$ chain, i.e. including arbitrary impurities. Both extensions were treated in a recent paper by Takebe [40]. It was shown that the 
generalized Bethe Ansatz of Takhtajan and Faddeev is applicable to this model. The corresponding $R$-matrix is given by

$$
R^{(12)}(u)=\left(\begin{array}{cccc}
a & 0 & 0 & d \\
0 & b & c & 0 \\
0 & c & b & 0 \\
d & 0 & 0 & a
\end{array}\right)
$$

in which

$a=\operatorname{sn}(u+2 \eta ; k) \quad, \quad b=\operatorname{sn}(u ; k) \quad, \quad c=\operatorname{sn}(2 \eta ; k) \quad, \quad d=k \operatorname{sn}(2 \eta ; k) \operatorname{sn}(u ; k) \operatorname{sn}(u+2 \eta ; k)$.

The quantum $L$-operator on each site $k$ of the spin chain now has the form

$$
L_{k}(u)=\left(\begin{array}{cc}
w_{0} \mathbf{1}+w_{3} \sigma_{k}^{3} & w_{1} \sigma_{k}^{1}-i w_{2} \sigma_{k}^{2} \\
w_{1} \sigma_{k}^{1}+i w_{2} \sigma_{k}^{2} & w_{0} \mathbf{1}-w_{3} \sigma_{k}^{3}
\end{array}\right)
$$

where

$$
\begin{gathered}
w_{0}+w_{3}=\operatorname{sn}(u+\eta ; k) \quad, \quad w_{0}-w_{3}=\operatorname{sn}(u-\eta ; k), \\
w_{1}+w_{2}=\operatorname{sn}(2 \eta ; k) \quad, \quad w_{1}-w_{2}=k \operatorname{sn}(2 \eta ; k) \operatorname{sn}(u+\eta ; k) \operatorname{sn}(u-\eta ; k) .
\end{gathered}
$$

The monodromy matrix takes again the form (6.4). The generalization of the algebraic Bethe Ansatz to the model described in (6.12), (6.13) is known only for the special eigenfunctions for which the number of excitations is half the number of spins in the chain. Of course, this constitutes only a special class within the total set of eigenfunctions, but no further results exist to date. Curiously, this is precisely the case in which we have a connection with the discrete RS model!

To be more precise, the generalized BA, developed in [39, 40], consists of constructing eigenfunctions of the trace of the monodromy matrix $A(u)+D(u)$ after performing a gauge transformation of the form

$$
T(u) \mapsto T_{m, m^{\prime}}(u)=S_{m}(u)^{-1} T(u) S_{m^{\prime}}(u)=:\left(\begin{array}{cc}
A_{m, m^{\prime}}(u) & B_{m, m^{\prime}}(u) \\
C_{m, m^{\prime}}(u) & D_{m, m^{\prime}}(u)
\end{array}\right)
$$

where $S_{m}(u)$ is an appropriate scalar matrix. (Note that we deviate from the notations in [39, 40] to be consistent with the notations used in earlier sections of the present paper.) 
Instead of a single vacuum there is now a set of generating (vacuum) vectors $\Omega_{M, n}$ for which one has

$$
A_{M, n}(u) \Omega_{M, n}=a(u) \Omega_{M, n-1} \quad, \quad D_{M, n}(u) \Omega_{M, n}=d(u) \Omega_{M, n+1} \quad, \quad C_{M, n}(u) \Omega_{M, n}=0 .
$$

The generalized Bethe eigenfunctions are linear combinations of the vectors

$$
\Psi_{n}\left(q_{1}, \ldots, q_{N}\right)=B_{n+1, n-1}\left(q_{1}\right) \ldots B_{n+N, n-N}\left(q_{N}\right) \Omega_{M, n-N}
$$

of the form

$$
\Psi_{\theta}\left(q_{1}, \ldots, q_{N}\right)=\sum_{n=-\infty}^{\infty} e^{2 \pi i n \theta} \Psi_{n}\left(q_{1}, \ldots, q_{N}\right)
$$

where $\theta$ is a free parameter. As it was pointed out in [39], it is possible that the series (6.17) is summable to zero for all $\theta$ except for a finite number of values $\theta_{j}$. (The results of Baxter [36] show that among the $\theta_{j}$ there is also the value $\theta=0$.) Then for such values of $\theta$ one can produce the corresponding eigenfunctions, provided that the excitation numbers $q_{1}, \ldots, q_{N}$ obey the following BAE's (cf. [40])

$$
\begin{gathered}
X \prod_{\substack{k=1 \\
k \neq \ell}}^{N} \frac{\sigma\left(q_{\ell}-q_{k}+2 \eta\right)}{\sigma\left(q_{\ell}-q_{k}-2 \eta\right)}=\prod_{k=1}^{2 N} \frac{\sigma\left(q_{\ell}-\delta_{k}+2 \eta\right)}{\sigma\left(q_{\ell}-\delta_{k}\right)}, \quad \ell=1, \ldots, N \\
X=\exp \left[-4 \pi i \theta+2 \eta \frac{\eta_{2}}{\omega_{2}}\left(\sum_{k=1}^{N}\left(2 q_{k}+2 \eta\right)-\sum_{k=1}^{2 N} \delta_{k}\right)\right]
\end{gathered}
$$

in the special case that $M=2 N$, and re-expressing the equations derived in 40 in terms of $\sigma$-function. Eqs. (6.18) correspond to the equations (2.14) for the discrete RS model in the elliptic case by the following identifications: $\lambda=2 \eta, \underset{q_{k}}{q_{k}}=\delta_{k}, \quad \widetilde{q}_{k}=\delta_{k+N}-2 \eta$, $(k=1, \ldots, N)$, together with

$$
\underset{\sim}{\frac{p}{p}}=\exp \left[-4 \pi i \theta-\lambda \frac{\eta_{2}}{\omega_{2}}(\underset{\sim}{B}+\widetilde{B}-2 B)\right], \quad B=\sum_{k=1}^{N} q_{k} .
$$

The sum of the particle positions $B$ plays the role of the boost generator of the underlying Poincaré algebra, cf. [10]. So, the generalized Bethe Ansatz equations correspond to the discrete-time system with the dynamics of $p$ given in terms of the dynamics of $q_{k}$ 's. The

${ }^{2}$ The relation $M=2 N$ follows because of spin- $\frac{1}{2}$ situation, while becoming $N=\ell M$ for the arbitrary $\operatorname{spin} \ell$ (cf. 40]). 
results of the Section 3 can be generalized to incorporate this case. In fact, we need to add now the term

$$
4 \pi i \theta B-\frac{\eta_{2}}{2 \omega_{2}} \lambda(\widetilde{B}-B)^{2},
$$

to the Lagrangian (3.1b) in order to get the correct factor from the Euler-Lagrange equations. If we now calculate the canonical momenta from $(3.1 \mathrm{~b})$ we get an extra term $-\frac{\eta_{2}}{\omega_{2}} \lambda(\widetilde{B}-B)$ on the right-hand side in (3.4), but this factor cancels again when we express the $h_{\ell}^{2}$ in terms of the canonical momenta, because of the $p$ entering into the eqs. (2.13). Thus, the final result (3.6) remains unaltered[. Consequently, we still have the same integrals in terms of the canonical variables, and the Ruijsenaars variables do not change, hence they are in involution and we have the integrable correspondence.

It is amazing that exactly in the restricted case where the generalized BA is known to apply, i.e. the case that the number of impurities is equal to twice the number of excitations, we have a connection with an integrable correspondence of RS type. This might also suggest that there exists a deeper relation between integrable quantum models solvable by the QISM on the one hand and classical integrable multiparticle models on the other hand. The connection provided here might yield an explanation for the existence conditions of the generalized BA of Faddeev and Takhtajan and of Baxter's solution of the eight-vertex model.

\section{Discussion}

The connection exhibited in the previous section between the BAE's for the XXZ and XYZ model with impurities and the discrete relativistic multi-particle model is very remarkable. A similar connection seems to exist also in the non-relativistic case studied in [1], 2], which yields a link between the equations of motion of the discrete CM model and BAE's for the Gaudin model. In both cases, we thus obtain a new interpretation of the BAE's for a quantum solvable model, namely as a classical integrable dynamical model with discrete time. Such interpretations, together with the exact solutions of the discrete equations of motion that were derived in section 4, might lead to possible ways of 'solving' the

\footnotetext{
${ }^{3}$ This effect can be viewed as a consequence of the relativistic invariance of the model.
} 
BAE's, (which in principle constitutes a set of transcendental equations), without having to perform some thermodynamic limit, cf. [38]. This, however, requires a reformulation of the initial value problem for the discrete model rather as a boundary value problem on the discrete-time chain. Although it is not clear at this stage that such boundary value problems are explicitely solvable as it was the case in Section 4, we nevertheless conjecture that this intriguing dynamical interpretation of the Bethe Ansatz equations will shed new light on the solvability of the corresponding quantum models. Furthermore, it would be interesting to study generalizations of our results, for instance in the directions of finding discrete particle models associated with higher-spin models and higher-rank associated Lie algebras (nested Bethe Ansatz), as well as investigating in this light the quadratic algebras associated with boundary conditions for quantum integrable systems (reflection equation algebras), cf. 433. The latter might lead to time-discretizations of $B C_{N}$ type of RS models, which were investigated in [14, 15].

\section{Acknowledgement}

FWN would like to thank the members of the Dipartimento di Fisica E. Amaldi of the Università di Roma III for their hospitality during a visit where this work was completed. He would also like to thank the Alexander von Humboldt Foundation for financial support at an earlier stage of the work, and especially Prof. B. Fuchssteiner for his hospitality during his stay at the University of Paderborn. Furthermore, he would like to express his great indebtness to Dr. G.D. Pang, as this work is a natural sequel to results obtained in collaboration with him. 


\section{References}

[1] F.W. Nijhoff and G.D. Pang, A time-discretized version of the Calogero-Moser model, Phys. Lett. 191A (1994) 101-107.

[2] F.W. Nijhoff and G.D. Pang, Integrable discrete-time particle systems and elliptic solutions of the lattice KP equations, in preparation.

[3] F.W. Nijhoff and G.D. Pang, Discrete-time Calogero-Moser model and lattice KP equations, in Proc. of the Intl. Workshop on Symmetries and Integrability of Difference Equations, eds. D. Levi, L. Vinet and P. Winternitz, (CRM Lecture Notes and Proceedings, to be published), hep-th/9409071.

[4] F. Calogero, Solution of the one-dimensional N-body problems with quadratic and/or inversely quadratic pair potentials, J. Math. Phys. 12 (1971), 419-436; J. Math. Phys. 10 (1969) 2191, ibid. 12 (1971) 418.

[5] B. Sutherland, Exact results for a quantum many-body problem in one dimension, Phys. Rev. A4 (1971) 2019; ibid. II, Phys. Rev. A5 (1972) 1372.

[6] J. Moser, Three integrable Hamiltonian systems connected with isospectral deformations, Adv. Math. 16 (1975), 197-220.

[7] M.A. Olshanetsky and A.M. Perelomov, Classical integrable finite-dimensional systems related to Lie algebras, Phys. Rep. 71 (1981), 313-400; Quantum integrable systems related to Lie algebras, ibid. 94 (1983), 313-404.

[8] A.P. Veselov, Integrable maps, Russ. Math. Surv. 46:5 (1991), 1-51.

[9] S. Wojchiechowski, The analogue of the Bäcklund transformation for integrable manybody systems, J. Phys. A: Math. Gen. 15 (1982) L653-657.

[10] S.N.M. Ruijsenaars and H. Schneider, A new class of integrable systems and its relation to solitons, Ann. Phys. 170 (1986) 370-405.

[11] S.N.M. Ruijsenaars, Finite-dimensional soliton systems, in Integrable and SuperIntegrable Systems, B. Kupershmidt, ed., (World Scientific Publ. Co., Signapore, 1990), pp. 165-206. 
[12] S.N.M. Ruijsenaars, Complete integrability of relativistic Calogero-Moser systems and elliptic function identities, Comm. Math. Phys. 110 (1987) 191-213.

[13] M. Bruschi and F. Calogero, The Lax representation for an integrable class of relativistic dynamical systems, Commun. Math. Phys. 109 (1987), 481-492.

[14] J.-F. van Diejen, Families of commuting difference operators, PhD Thesis, University of Amsterdam, (June, 1994).

[15] T.H. Koornwinder, Askey-Wilson polynomials for root systems of type BC, in: Hypergeometric functions on domains of positivity, Jack polynomials, and applications, ed. D.St.P. Richards, Contemp. Math. 138 (1992), pp. 189-204.

[16] B. Feigin, E. Frenkel, and N. Reshetikhin, Gaudin model, Bethe ansatz and correlation functions at the critical level, hep-th/9402022.

[17] P. I. Etingof, Quantum integrable systems and representations of Lie algebras, hepth/9311132.

[18] I. Cherednik, Integration of quantum many-body problems by affine KnizhnikZamolodchikov equations, Adv. Math. 106 (1994) 69-95.

[19] M. Bruschi, O. Ragnisco, P.M. Santini and G.-Z. Tu, Integrable symplectic maps, Physica 49D (1991), 273-294.

[20] A. Erdelyi e.a., Higher Transcendental Functions, volume 2, (McGraw-Hill, New York, 1953).

[21] G. Frobenius, Über die elliptischen Funktionen zweier Art, J. Reine Angew. Math. 93 (1882) 53-68.

[22] E.K. Sklyanin, On the poisson structure of the periodic classical XYZ-chain, J. Sov. Math. 46 (1989) 1664-1683. [Zap. Nauch. Sem. 150 (1986) 154-180.]

[23] E.T. Whittaker and G.N. Watson, A Course in Modern Analysis, (Cambridge University Press, 4th ed., 1988). 
[24] F.W. Nijhoff, V.G. Papageorgiou and H.W. Capel, Integrable time-discrete systems: lattices and mappings, in: Quantum Groups, ed. P.P. Kulish, Springer LNM 1510 (1992), pp. $312-325$.

[25] A.P. Veselov, Growth and integrability in the dynamics of maps, Commun. Math. Phys. 145 (1992) 181-193.

[26] H. Airault, H.McKean, and J. Moser, Rational and elliptic solutions of the Kortewegde Vries equation and a related many-body problem, Commun. Pure Appl. Math., 30 (1977), 95-148.

[27] D.V. Chudnovsky and G.V. Chudnovsky, Pole expansions of nonlinear partial differential equations, Nuovo Cim. 40B (1977), 339-353.

[28] I. M. Krichever, Rational solutions of the Kadomtsev-Petviashvili equation and integrable systems of $N$ particles on a line, Funct. Anal. Appl., 12 (1978), 59-61.

[29] O. Babelon and D. Bernard, The sine-Gordon solitons as a N-body problem, Preprint SPhT-93-072, LPTHE-93-40, hep-th/9309154.

[30] F.W. Nijhoff, H.W. Capel, G.L. Wiersma and G.R.W. Quispel, Bäcklund transformations and three-dimensional lattice equations, Phys. Lett. 105A (1984), 267-272.

[31] F.W. Nijhoff, H. Capel and G.L. Wiersma, Integrable lattice systems in two and three dimensions, in Geometric Aspects of the Einstein Equations and Integrable Systems, ed. R. Martini, Springer Lect. Notes Phys. 239 (1985), pp. 263-302.

[32] F.W. Nijhoff, V.G. Papageorgiou, H.W. Capel and G.R.W. Quispel, The lattice Gel'fand-Dikii hierarchy, Inv. Probl. 8 (1992) 597-621.

[33] H.W. Capel and F.W. Nijhoff, Integrable lattice equations, in: Important Developments in Soliton Theory, eds. A.S. Fokas and V.E. Zakharov, (Springer Lect. Notes in Nonlinear Dynamics, 1993), pp. 38-57.

[34] R. Hirota, Discrete analogue of generalized Toda equation, J. Phys. Soc. Japan 50 (1981) 3785-3791. 
[35] E. Date, M. Jimbo and T. Miwa, Method for generating discrete soliton equations II, J. Phys. Soc. Japan 51 (1982) 4125-4131.

[36] R.J. Baxter, Exactly Solved models in Statistical Mechanics, (Academic Press, London, 1982).

[37] L.D. Faddeev, Quantum Inverse Scattering Method, Sov. Sci. Revs. C1 (1981) 107160.

[38] V.E. Korepin, N.M. Bogoliubov and A.G. Izergin, Correlation Functions for Integrable Systems and Quantum Inverse Scattering Method, (Academic Press, New York, 1994).

[39] L.A. Takhtadzhan and L.D. Faddeev, The Quantum Method of the Inverse Problem and the Heisenberg XYZ Model, Russ. Math. Surv. 34 \# 5 (1979) 11-68.

[40] T. Takebe, Generalized Bethe ansatz with the general spin representations of the Sklyanin algebra, J. Phys. A: Math. Gen. 25 (1992) 1071-1083.

[41] L.D. Faddeev, Algebraic Aspects of Bethe-Ansatz, Preprint ITP-SB-94-11, (March 1994), hep-th/9404013.

[42] E. K. Sklyanin, Some algebraic structures connected with the Yang-Baxter equation, Funct. Anal. Appl. 16 (1983) 263-270; ibid. II Representations of quantum algebras, Funct. Anal. Appl. 17 (1984) 273-284.

[43] E.K. Sklyanin, Boundary conditions for quantum integrable systems, J. Phys. A: Math. Gen. 21 (1988) 2375-2387. 